\title{
Serum Neutrophil/Lymphocyte Ratio and Platelet/Lymphocyte Ratio before and after Thrombolysis in Cerebral Infarction
}

\author{
Chunjie Zhang ${ }^{1,2}$, Xiaohong $\mathrm{Li}^{1,2}$
}

\author{
${ }^{1}$ Weifang Medical University, Weifang, Shandong, China \\ ${ }^{2}$ Jinan Central Hospital, Jinan, Shandong, China
}

\begin{abstract}
Objective To investigate the changes of serum neutrophil/lymphocyte ratio (NLR) and platelet/lymphocyte ratio (PLR) before and after thrombolysis in acute cerebral infarction (ACI) and their clinical significance. Methods A total of 100 patients diagnosed with acute cerebral infarction and undergoing intravenous thrombolysis in the Department of Neurology of Jinan Central Hospital from January 2019 to October 2019 were selected and divided into the mild functional impairment group according to the neurological impairment score (NIHSS score) ( $\leq 12$ points) and severe functional impairment group ( $>12$ points)), retrospectively analyze the patient's general clinical data and biochemical indicators such as neutrophils (NC), platelets (PLT) and lymphocytes (LC), and calculate NLR , PLR. Compare the changes of NLR and PLR between different groups. Results The levels of NLR and PLR in the severe functional impairment group before thrombolysis were significantly higher than those in the mild functional impairment group. The levels of NLR and PLR in the severe functional impairment group after thrombolysis were significantly higher than those in the mild functional impairment group. The difference was statistically significant. Conclusion Serum NLR and PLR are related to the severity of ACI.
\end{abstract}

Keywords: Cerebral Infarction, Neutrophil / Lymphocyte Ratio, Platelet / Lymphocyte Ratio, Thrombolytic Therapy

\section{Introduction}

Acute cerebral infarction (ACI), also known as acute ischemic stroke, is a disorder of blood supply to the brain caused by various reasons, causing cerebral ischemia and hypoxia, leading to local avascular necrosis and softening of brain tissue. The incidence rate accounts for more than $60 \%$ of stroke [1], and it is currently one of the main diseases that cause disability and death in humans. Atherosclerosis is the most important pathogenic factor of ischemic stroke, which is mainly caused by stenosis of blood vessel lumen caused by atherosclerotic plaque or rupture of cerebral atherosclerotic plaque. Relevant studies have shown that atherosclerosis is essentially an inflammatory process. The blood vessel wall of the body is affected by a variety of physical and chemical factors, resulting in the deposition of inflammation-mediated lipids on the blood vessel wall, thereby forming atherosclerotic plaques. Inflammatory mediators Play an important role in the entire course of atherosclerosis [2]. At the same time, various mediators can appear when blood vessels rupture and activate platelets to produce blood clots. Studies have shown that the level of platelet activation in patients with acute cerebral infarction can be significantly increased, and the change pattern of platelets and their related activation indicators is of great significance for the diagnosis and treatment of acute cerebral infarction [3]. Endovascular therapy, brain protection therapy and antithrombotic therapy are typical acute treatments. Relevant studies have shown that intravenous t-PA treatment is strongly recommended for acute cerebral infarction [4]. This study analyzed 100 patients with acute cerebral infarction with thrombolytic NLR and PLR, explored the relationship between NLR, PLR and the severity of ACI lesions, and analyzed their role in acute cerebral infarction.
Materials and methods
1. General information
A retrospective analysis of 100 patients with acute cerebral infarction in the Department of Neurology of Jinan Central Hospital from January 2019 to October 2019. All patients met the diagnostic criteria of acute cerebral infarction in the 2018 Chinese Acute Ischemic Stroke Diagnosis and Treatment Guidelines[ 5]
. Among them, male cases and female cases.
2. Selection criteria
(1)Age $18-80$ years; (2) Onset time is within 4.5 hours; (3) Neural damage lasts for more than 1 hour; (4) Head CT except cerebral hemorrhage; 5 Excluding large area cerebral infarction on MRI DWI; (6) The neurological deficit score is $5-25$ points (7) The patient and family members agree and sign the thrombolytic treatment consent form.
3.Exclusion criteria
(1) Have a history of brain trauma, cerebral infarction, and myocardial infarction within the past 3 months, excluding those who have no clinical symptoms in old lacunar infarction; (2) Have gastrointestinal or urinary 
Serum Neutrophil/Lymphocyte Ratio and Platelet/Lymphocyte Ratio before and after Thrombolysis in Cerebral Infarction

tract bleeding within the past 3 weeks; (3) In the past 2 weeks Major surgical operation; (4)Arterial puncture at a site that is not easy to be compressed within the past 1 week; 5) Active bleeding or trauma is found during physical examination; (6) Previous cerebral hemorrhage, subarachnoid hemorrhage, mixed stroke; (7) Severe heart disease Liver, kidney disease and severe diabetes; (8) unconsciousness; (9) blood pressure over 180/100 $\mathrm{mmHg}, \mathrm{INR}>1.5$; 10 pregnancy or menstrual period.

4. Grouping method

According to the National Institutes of Health Stroke Neurological Impairment Score Scale (NIHSS), the patients were scored according to the degree of damage before thrombolysis. The score $\leq 12$ was defined as the mild functional impairment group, and the score $>12$ was defined as the severe Functional impairment group.

5. Treatment methods

Patients with acute cerebral infarction were given rt-PA (Shanghai Boehringer Ingelheim Pharmaceutical Co., Ltd., specification: $50 \mathrm{mg}$, batch number $=002735$ ) intravenous ultra-early thrombolytic therapy within 4.5 hours of onset, and use $0.9 \mathrm{mg} / \mathrm{kg}$ body weight according to the instructions If the total amount does not exceed $90 \mathrm{mg}, 10 \%$ of the drug is injected intravenously within 1 minute, and the remaining $90 \%$ is slowly injected intravenously within 1 hour.

6. Record indicators

Record the relevant laboratory indicators before and after thrombolysis: routine blood and blood coagulation routine. The above indicators were measured by the laboratory physicians of our hospital with the automatic biochemical detector from Hitachi. Related imaging findings: MRI+MRA, bilateral carotid artery ultrasound.

7. Statistical analysis

Using SPSS21.0 software, measurement data were expressed as mean $\pm \mathrm{SD}$ deviation $(\mathrm{x} \pm \mathrm{s})$, the comparison of means between the two groups was performed by $t$ test; the correlation between DPN and each clinical index was analyzed by multivariate logistic regression analysis. $\mathrm{P}<0.05$ means the difference is statistically significant.

\section{Results}

Comparison of general information and biochemical indicators

There was no significant difference in age, gender, and past history between the two groups of patients $(\mathrm{P}>0.05)$. The levels of NLR and PLR before thrombolysis in the severe functional impairment group $(136.77 \pm 57.06)$ were significant in the mild functional impairment group $(111.57 \pm 38.25)$, and the difference was statistically significant $(\mathrm{P}<0.05)$. The levels of NLR and PLR in the severe functional impairment group (136.77 \pm 57.06$)$ were significantly changed in the mild functional impairment group (111.57 \pm 38.25$)$, and the difference was statistically significant $(\mathrm{P}<0.05)$.

\section{Discussion}

Cerebral infarction is recognized as a serious global health problem due to its high morbidity, high disability, high mortality, and high medical expenses. The incidence of cerebral infarction has increased with the increase of Chinese elderly. The treatment of patients with cerebral infarction is mainly early intravenous thrombolytic therapy and later conventional drug treatment. Early detection and early treatment can greatly reduce the disability rate of cerebral infarction. Therefore, early intravenous thrombolysis is very important for patients with acute cerebral infarction. After intravenous thrombolysis, the main indicator of neurological function recovery in patients is the evaluation of neurological deficit score (NIHSS score). Clinical manifestations evaluate the scores of patients. Some patients may have repetitive symptoms and unobvious symptoms, which may interfere with the evaluation work of clinicians, and thus the evaluation scores will be biased, which may interfere with the understanding of the patient's neurological impairment. Therefore, it is necessary for clinicians to use objective laboratory data to evaluate the degree of nerve damage in patients.

The main cause of cerebral infarction is atherosclerosis. More and more studies have shown that atherosclerosis is an inflammatory process of chronic vascular injury, and inflammatory mediators play an important role in the entire course of atherosclerosis. At the same time, inflammatory mediators induce thrombocytosis by stimulating megakaryocytes. Therefore, some people think that the increase in platelet level is a sign of prethrombotic activity and stop. The level of lymphocytes is also affected by the inflammatory state. Lymphopenia is due to the acceleration of apoptosis due to the systemic inflammation process, so lymphocytes have a regulatory effect on controlling inflammation [6]. Related experiments have shown that after cerebral infarction, the local brain tissue rapidly initiates an inflammatory response under the stimulation of ischemia and hypoxia, and its degree and scope are related to the infarct volume and the severity of infarct symptoms; if intravenous thrombolysis is given in a $4.5 \mathrm{~h}$ time window in time Treatment and rescue of ischemic penumbra may interrupt the inflammatory response of some tissues. Due to its high stability, PLR has been studied in various inflammations, tumors, cardiovascular diseases, etc., but the relationship between NLR, PLR and the severity of ACI lesions has not been reported yet.

This study showed that the changes in the levels of NLR and PLR in the severe functional impairment group were significantly higher than those in the mild functional impairment group, while the levels of NLR and PLR before thrombolysis in the severe injury group were significantly higher than those in the mild injury group, indicating that NLR and PLR in patients with severe acute cerebral infarction It is expressed earlier, 
Serum Neutrophil/Lymphocyte Ratio and Platelet/Lymphocyte Ratio before and after Thrombolysis in Cerebral Infarction

and thrombolytic therapy is performed early to restore the ischemic penumbra, which may reduce the inflammatory response. PLR can be calculated from blood routines and blood coagulation routines. It is simple and easy to operate for patients with acute cerebral infarction, with less trauma and low cost. Therefore, clinical biochemical indicators can be used to predict the degree of nerve damage and the effect of thrombolytic therapy in patients. In summary, patients with cerebral infarction thrombolytic therapy will become more and more aware of cerebral infarction with the public, and the changes in NLR and PLR levels can be used to understand the neurological impairment of patients.

\section{Reference}

1. Camerlingo M, Valente L, Tognozzi M, et al. C-reactive protein levels in the first three hours after acute cerebral infarction.
International Journal of Neuroscience, 2011, 121 (2) :65-68.

2. $\mathrm{Li} \mathrm{Bo}$, Chen Yundai. Research progress on the effect of glucagon-like peptide-1 on atherosclerosis[ $\mathrm{J}$ ]. Chinese Journal of Arteriosclerosis, 2017, 25(04), 427-432.

3. Shi Lei. The relationship between the severity of acute cerebral infarction and the activation and aggregation of platelets and its clinical significance [J]. China Modern Medicine Application, 2016, 10 (3): 24-25.

4. Shibata K, Hashimoto T, Miyazaki T, Miyazaki A, Nobe K .CurrPharmDes. Thrombolytic Therapy for Acute Ischemic Stroke: Past and Future.2019;25(3):242-250. doi: 10.2174/1381612825666190319115018.

5. Neurology Branch of Chinese Medical Association. Cerebrovascular Disease Group of Neurology Branch of Chinese Medical Association. Guidelines for Diagnosis and Treatment of Acute Ischemic Stroke in China 2018. Chinese Journal of Neurology. 2018, 51(9): 666-682.

6. Liu Li, Xie Xiaona, Li Hongyan, et al. Research progress on the correlation between peripheral blood white blood cell count and diabetes and its complications[J]. Chinese General Practice, 2012(11): 115-116 